\title{
Detachment Dynamics of Graphene Nanoribbons on Gold
}

Lorenzo Gigli, ${ }^{\dagger \dagger}$ Shigeki Kawai, ${ }^{*} \neq \odot$ Roberto Guerra, ${ }^{\S, \|_{\odot}}$ Nicola Manini, ${ }^{\S}$ Rémy Pawlak, ${ }^{\perp}{ }_{\odot}$

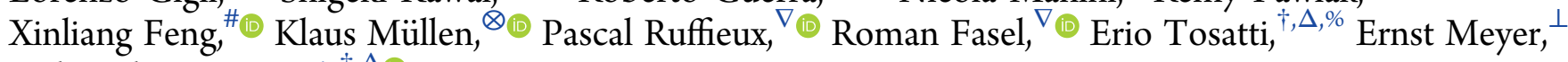
and Andrea Vanossi ${ }^{*}, \dagger, \Delta$

\author{
${ }^{\dagger}$ International School for Advanced Studies (SISSA), Via Bonomea 265, 34136 Trieste, Italy \\ ${ }^{\ddagger}$ International Center for Materials Nanoarchitectonics, National Institute for Materials Science, 1-1, Namiki, Tsukuba, Ibaraki \\ 305-0044, Japan \\ ${ }^{\S}$ Dipartimento di Fisica, Università degli Studi di Milano, Via Celoria 16, 20133 Milano, Italy \\ "Center for Complexity and Biosystems, University of Milan, 20133 Milan, Italy \\ ${ }^{\perp}$ Department of Physics, University of Basel, Klingelbergstr. 82, CH-4056 Basel, Switzerland \\ \# Department of Chemistry and Food Chemistry, Center for Advancing Electronics Dresden (CFAED), Technische Universität \\ Dresden, 01062 Dresden, Germany \\ ${ }^{\otimes}$ Max Planck Institute for Polymer Research, 55124 Mainz, Germany

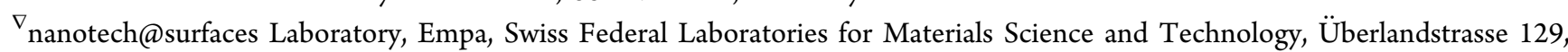 \\ 8600 Dübendorf, Switzerland \\ ${ }^{\Delta}$ CNR-IOM Democritos National Simulation Center, Via Bonomea 265, 34136 Trieste, Italy \\ ${ }^{\%}$ The Abdus Salam International Centre for Theoretical Physics (ICTP), Strada Costiera 11, 34151 Trieste, Italy
}

\section{Supporting Information}

\begin{abstract}
Metal-surface physisorbed graphene nanoribbons (GNRs) constitute mobile nanocontacts whose interest is simultaneously mechanical, electronic, and tribological. Previous work showed that GNRs adsorbed on $\mathrm{Au}(111)$ generally slide smoothly and superlubrically owing to the incommensurability of their structures. We address here the nanomechanics of detachment, as realized when one end is picked up and lifted by an AFM cantilever. AFM nanomanipulations and molecular-dynamics (MD) simulations identify two successive regimes, characterized by (i) a progressively increasing local bending, accompanied by the smooth sliding of the adhered part, followed by (ii) a stick-slip dynamics involving sudden bending relaxation associated with intermittent jumps of the remaining adhered GNR segment and tail end. AFM measurements of the vertical force exhibit oscillations which, compared with MD simulations, can be associated with the successive detachment of individual GNR unit cells of length $0.42 \mathrm{~nm}$. Extra modulations within one single period are caused by steplike advancements of the stillphysisorbed part of the GNR. The sliding of the incommensurate moire pattern that accompanies the GNR lifting generally yields an additional long-period oscillation: while almost undetectable when the GNR is aligned in the standard "R30" orientation on $\mathrm{Au}(111)$, we predict that such feature should become prominent in the alternative rotated "R0" orientation on the same surface, or on a different surface, such as perhaps $\operatorname{Ag}(111)$.
\end{abstract}

KEYWORDS: graphene nanoribbons, nanocontacts, manipulation, nanomechanics, moiré pattern

U nderstanding the adhesive and frictional properties of nanosystems, such as molecules, 3D nanoclusters or 2D adsorbates, polymeric chains, etc., on structurally well-characterized crystalline substrates ${ }^{1-18}$ is of key importance for fundamental sciences such as contact mechanics and nanotribology and for technological applications. Indeed, controlled nanomanipulation of deposited nano-objects, in terms of positioning, adhesion, depinning, and sliding, can be used to build molecular superstructures, to explore the influence of the environment on individual molecules, or to perform engineering operations at the ultimate, molecular, limit of fabrication for hi-tech nanodevices.

Mostly due to the extremely large surface-to-volume ratio, the peculiar behavior of these nanosystems may present

Received: October 16, 2018

Accepted: December 10, 2018

Published: December 10, 2018 

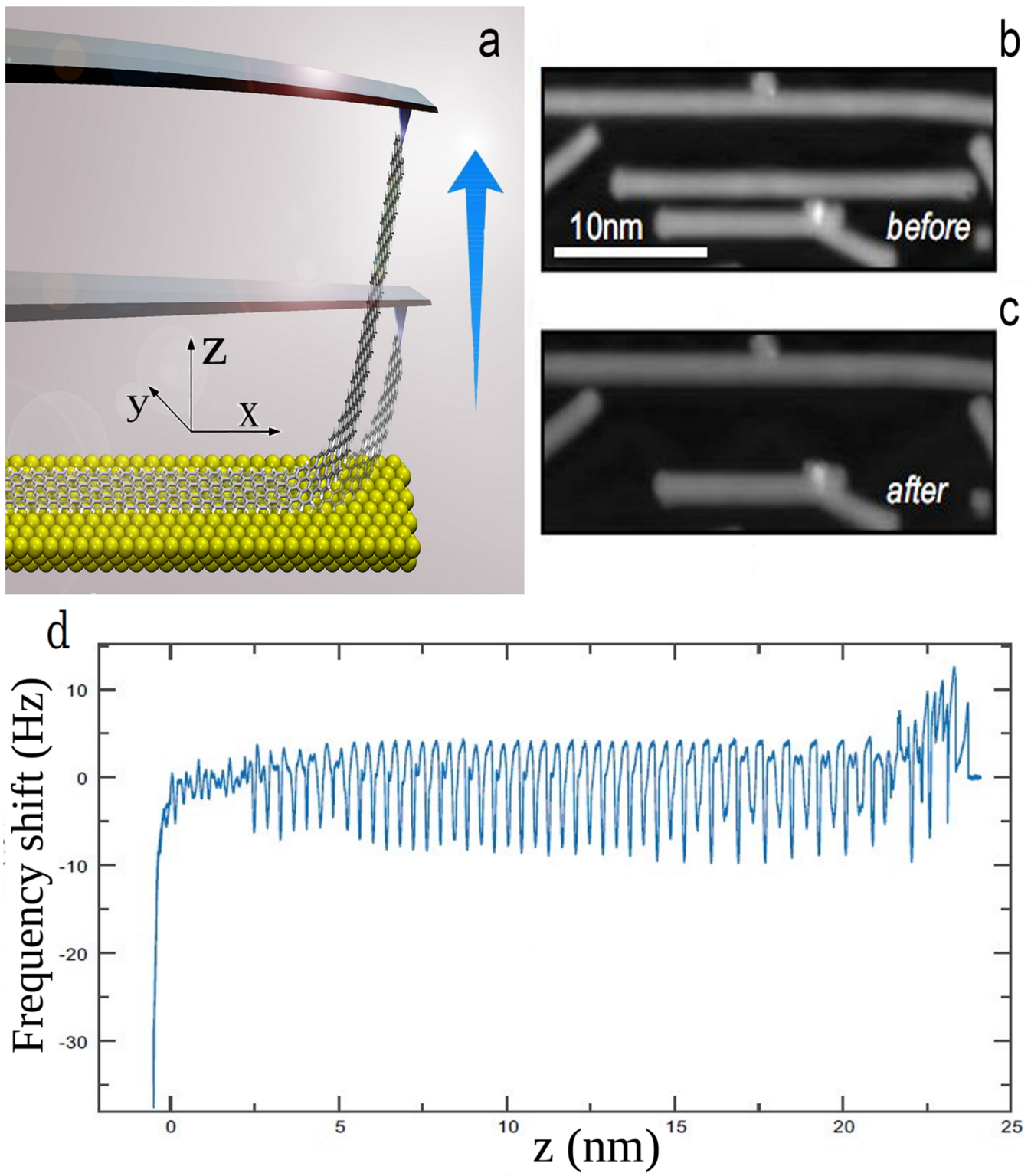

Figure 1. (a) Sketch of the tip-GNR setup in two distinct configurations of the GNR during detachment. (b, c) Two STM scans taken before and after detachment. (d) Experimental frequency-shift trace recorded as the GNR is lifted up until detachment.

properties that vary dramatically with size. Atomically precise synthesis techniques, ${ }^{19,20}$ experimentally tuning their physical and chemical characteristics during the preparation procedure, may assemble structurally similar geometries, with quite distinctive features influencing the system in terms of adhesive and friction-related response.

With their strong resilient structure and the experimental possibility to be picked up at one edge, dragged laterally and/ or lifted up vertically by atomic force microscopy (AFM) techniques, graphene nanoribbons (GNRs) physisorbed on $\mathrm{Au}(111)$ surfaces do represent an important actor in this exploration.

AFM-driven sliding displacements have been already exploited to probe the frictional properties of this interface at very low temperature, ${ }^{8}$ providing initial evidence for a weak length dependence of the static friction of armchair nanoribbons. Paralleling those experimental results, subsequent numerical simulations ${ }^{12}$ have confirmed a frictionally superlubric regime characterized by small friction that is strongly oscillating and basically periodic with no average increase upon increase of the GNR length. With a static friction trend dictated by the characteristic periodicity of the graphene-gold interface moire pattern, the simulated dissipative behavior already highlighted the occurrence of different dynamical regimes, ranging from smooth sliding to multiple stick-slip friction depending on the vertical height of the lifted, and laterally pulled, end. ${ }^{13}$

In the present work, by means of nonequilibrium atomistic MD simulations and AFM manipulation data, we investigate the detailed mechanisms of detachment of a $30 \mathrm{~nm}$ long armchair GNR from the $\mathrm{Au}(111)$ surface upon vertical lifting of one end. Depending on the actual configuration at the nanoribbon/gold interface, the combined theoretical and experimental analysis sheds light, at a molecular level, on the manner in which adhesion and lateral corrugation of the nanocontact determine the characteristic periodicities observed in the lifting force and its $z$-derivative during the progressive detachment process upon lifting. 


\section{RESULTS AND DISCUSSION}

Detachment Dynamics at R30. Experiment. Seven carbon atoms wide $(n=7)$ armchair GNRs are synthesized on a clean $\mathrm{Au}(111)$ surface at $4.8 \mathrm{~K}$ in $\mathrm{UHV}$, as detailed in the Methods. We control the Au-covered AFM tip so that it picks up a $\sim 25 \mathrm{~nm}$ long GNR at one end, producing unilateral detachment as sketched in Figure 1a. Figure 1b shows a STM topography of the surface before performing the manipulation of that GNR aligned along the $[-1,0,1]$ direction of the gold substrate, also called R30. This is, within the herringbone reconstruction of $\mathrm{Au}(111)$, the spontaneous orientation adopted by the GNR. In the specific case, the GNR crosses two fcc-hcp terraces of the gold substrate, as highlighted in Figure S1. The tip is positioned at one GNR end, with a low bias voltage $(\sim 2 \mathrm{mV})$. When the junction between the tip and the GNR is established, an abrupt increase in the tunneling current is detected. Subsequently, a dynamical AFM mode is turned on, with a vertical oscillation amplitude $\simeq 43 \mathrm{pm}$. The tip is then slowly retracted while recording the frequency shift (Figure 1d) as well as the energy dissipation (see Figure S1). Figure 1c shows the STM topography taken after the full detachment of that GNR, followed by tip cleaning outside the scan area. Figure $1 \mathrm{~d}$ shows the measured frequency shift, i.e., the variation of the resonance frequency of the AFM force sensor upon vertical lifting of the GNR, as a function of the height $z$. This frequency shift is proportional to the derivative of the vertical force that the tip exerts on the GNR: $\mathrm{d} F_{\text {ext }} / \mathrm{d} z$. ${ }^{5}$ This force gradient measurement with a stiff cantilever realizes a high sensitivity measurement while avoiding a typical mechanical instability, which can be caused in the quasi-static force measurement with a soft cantilever. ${ }^{21,22}$ After an initial steep increase of the frequency shift, due to the GNR curvature build-up, an oscillating signal is observed, with a main periodicity $\simeq 0.40 \mathrm{~nm}$.

Simulation and Theory. In order to shed light on the physical mechanisms that come into play in the detachment dynamics, we simulate this same system with nonequilibrium molecular dynamics (NEMD). We construct an $n=7$ armchair GNR, consisting of a stripe of alternating triplets and pairs of carbon hexagons, of width $\simeq 0.7 \mathrm{~nm}$ and length $\simeq 30.2 \mathrm{~nm}$, where all the peripheral $\mathrm{C}$ atoms are passivated with hydrogens. ${ }^{12,13}$ All carbon atom coordinates are fully mobile. The $\mathrm{Au}(111)$ substrate consists of two unreconstructed layers, also fully mobile, on top of one rigid layer, with fcc stacking. The lifting effect of the AFM tip on the first row of three C atoms is simulated by means of a vertical spring with an effective elastic constant $k_{z}=1800 \mathrm{~N} / \mathrm{m}$. One end of this spring moves vertically with constant velocity $v_{0}=0.5 \mathrm{~m} / \mathrm{s}$ along the $z$-axis of Figure 1a. Although much larger than the experimental speed, $\simeq 1 \mu \mathrm{m} / \mathrm{s}$, we verified that the simulated lifting speed is still small enough to yield meaningful, speedindependent results. The spring is attached to the three $\mathrm{C}$ atoms in the first row. The in-plane coordinates $(x, y)$ of these three atoms are kept fixed during detachment, so that the lifting is vertical. The simulation proceeds until complete detachment of the GNR is achieved. To gain insight into the physics of this system, we are interested in comparing the timeevolution (or equivalently the evolution against the $z$ coordinate of the driving spring end) of the following quantities:

- the instantaneous vertical force acting along the pulling direction, namely $F_{\text {ext }}^{z}(t)=3 k_{z}\left[v_{0} t-z_{\text {end }}(t)\right]$, where $z_{\text {end }}(t)=\sum_{i=1}^{3} z_{i}(t) / 3$ is the average $z$-coordinate of the lifted end of the GNR;

- the vertical force gradient $\mathrm{d} F_{\text {ext }} / \mathrm{d} z$, directly comparable to measurements, which is proportional to the frequency shift $\delta f(z), \mathrm{d} F_{\text {ext }} / \mathrm{d} z=\kappa \delta f(z)$, where $\kappa=0.15 \mathrm{~N} \mathrm{~m}^{-1}$ $\mathrm{Hz}^{1-}$ is a conversion factor; ${ }^{8}$

- the deviations of the GNR-substrate total adhesive energy $\Delta E_{\text {adh }}(t)$ and the gold-gold total potential energy $\Delta E_{\mathrm{Au}-\mathrm{Au}}(t)$ away from their linear behavior $E(t)=E^{0}-P t$ where $P$ and $E^{0}$ are fitting parameters;

- the total variation of the intra-GNR carbon-carbon and carbon-hydrogen potential energy $\Delta E_{\mathrm{GNR}}(t)=$ $E_{\mathrm{GNR}}(t)-E_{\mathrm{GNR}}(0)$. The value at $t=0$ corresponds to an unlifted relaxed GNR on gold;

- the horizontal advancement of the trailing end of the GNR along the $x$-axis $\Delta x_{\text {tail }}(t)=x_{\text {tail }}(t)-x_{\text {tail }}(0)$. The quantity $x_{\text {tail }}(t)=\sum_{j=1}^{3} x_{j}(t) / 3$ is obtained by averaging the instantaneous $x$-coordinate of the end row of $\mathrm{C}$ atoms, the last ones to get lifted up at complete peel-off.

These physical observables are conveniently reported as functions of the dimensionless vertical displacement $z / a_{\mathrm{GNR}}$, where $a_{\mathrm{GNR}}=0.42 \mathrm{~nm}$ equals one GNR unit cell length, and the reference coordinate $z=0$ is taken at the average vertical position of the $\mathrm{C}$ atoms of the fully relaxed GNR on the substrate in the unlifted configuration.

The detachment dynamics of the GNR occurs following two successive regimes. In the first regime at low lifting for $z<3$ $\mathrm{nm}$, the vertical motion of the AFM initially builds up the GNR curvature, marked by an increase of the GNR bending energy, as shown in Figure S2. The bending energy rise is consistent with the initial upswing of the experimental frequency shift of Figure 1d. The GNR-Au(111) corrugation energy is small compared to the system bending elasticity, so that the first relevant, yet gradual, buildup of the GNR curvature results in a smooth sliding of the still attached GNR tail. For $z \simeq 3 \mathrm{~nm} \simeq 7 a_{\mathrm{GNR}}$ the frequency-shift profile reaches a plateau with a superimposed oscillation. This second regime corresponds to a steady peeling. Figure 2 shows in this second regime both the experimental and the theoretical force derivative curves. The simulated force derivative is smaller in magnitude and not identical in line shape to the experimental one. In that respect, one must recall that the force fluctuations represent only a small deviation relative to the total lifting force, a very large background quantity which is unaccessible experimentally but dominating in simulation.

The level of quantitative agreement between theory and experiment is limited by the use of simple two-body LennardJones potentials to describe simultaneously adhesive interactions and the interface corrugation. The specific values of the LJ parameters adopted in the present work represent the best possible compromise to reproduce at the same time the correct order of magnitude of the adhesive forces for carbon/metal interactions and the crucial features of the lifting dynamics associated with lateral corrugation.

First, the main periodicity is equal to the GNR unit length $a_{\mathrm{GNR}}$, consistent with the observation that the vertical motion of the spring induces a sequence of discrete detachments of GNR sections with the size of one GNR unit cell, until complete pull-off. Second, within one single period of this vertical lifting the detachment force experiences significant and roughly periodic drops, that occur in coincidence with steplike slips of the still-physisorbed section of the GNR, as illustrated 


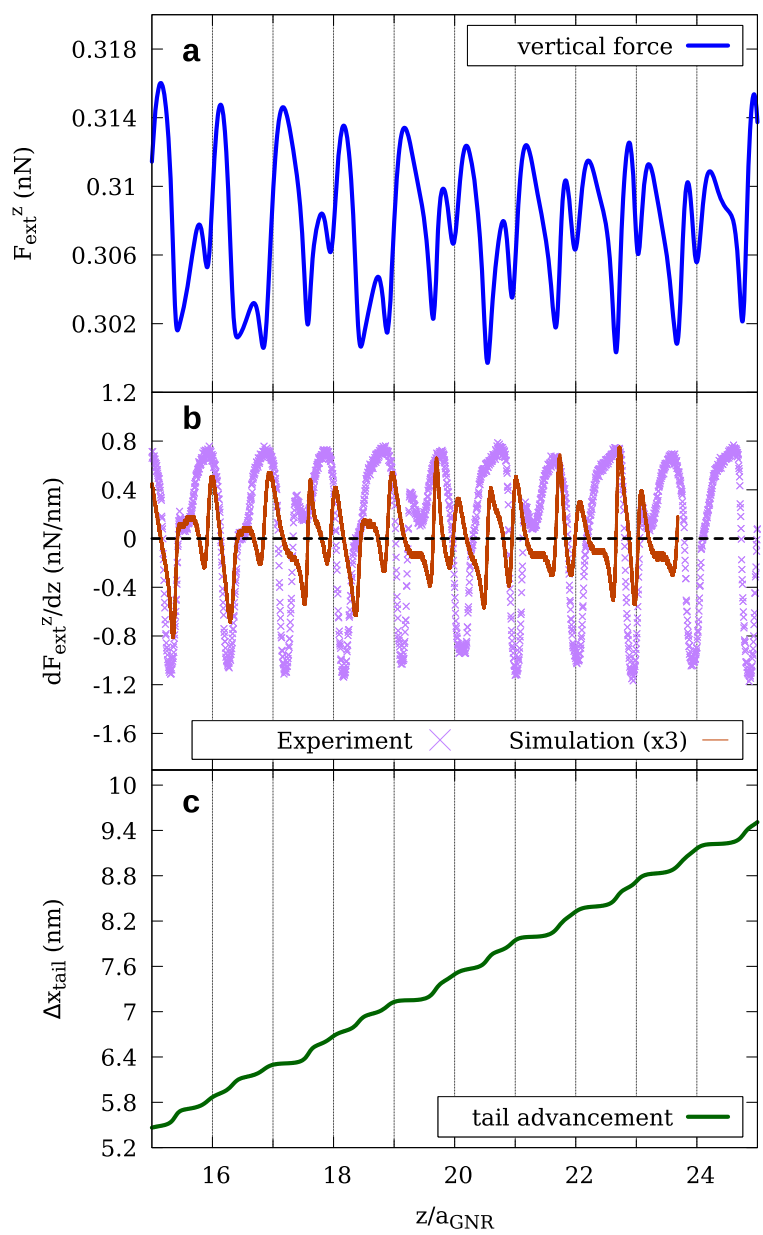

Figure 2. Detachment dynamics of the R30-oriented GNR/ $\mathrm{Au}(111)$ in the height range $15 a_{\mathrm{GNR}}<z<25 a_{\mathrm{GNR}}$. The simulated lifting speed is $v_{0}=0.5 \mathrm{~m} / \mathrm{s}$; the experimental speed is much smaller. Comparison of (a) the simulated external lifting force, (b) the experimental and the simulated force gradient magnified by three times, and (c) the tail end $x$-advancement. The lifting-height scale is expressed in units of $a_{\mathrm{GNR}}=0.42 \mathrm{~nm}$ corresponding to one unit cell length of the GNR (one triplet plus one doublet of carbon hexagons).

by small steps advancement of the tail in Figure $2 c$. We verified that the force drops are enhanced by the gold mobility by repeating the entire detachment simulation with a substrate consisting of gold atoms frozen in their crystalline arrangement. A direct comparison between the results of the simulations with the mobile and the rigid substrate is provided in Figure S3 of the Supporting Information. The importance of the gold mobility, an element not discussed before, is also confirmed by the behavior of the fluctuations of the gold-gold potential energy of Figure 3b: they are small but not entirely negligible compared to the variations of the other energy terms of the system.

Focusing on just four $a_{\mathrm{GNR}}$ lifting periods for heights $15 a_{\mathrm{GNR}}<z<19 a_{\mathrm{GNR}}$, Figure 3 provides further insight into the detachment. This zoomed-in detail shows that the main periodicity of $F_{\text {ext }}^{z}(t)$ corresponds to the detachment of a full GNR unit cell. As in the experiments, that main period is additionally decorated by superimposed oscillating features. These finer force derivative features do not arise, as one might have initially suspected, from the progressive detachment of the $n=7$ armchair GNR substructures (e.g., the alternating

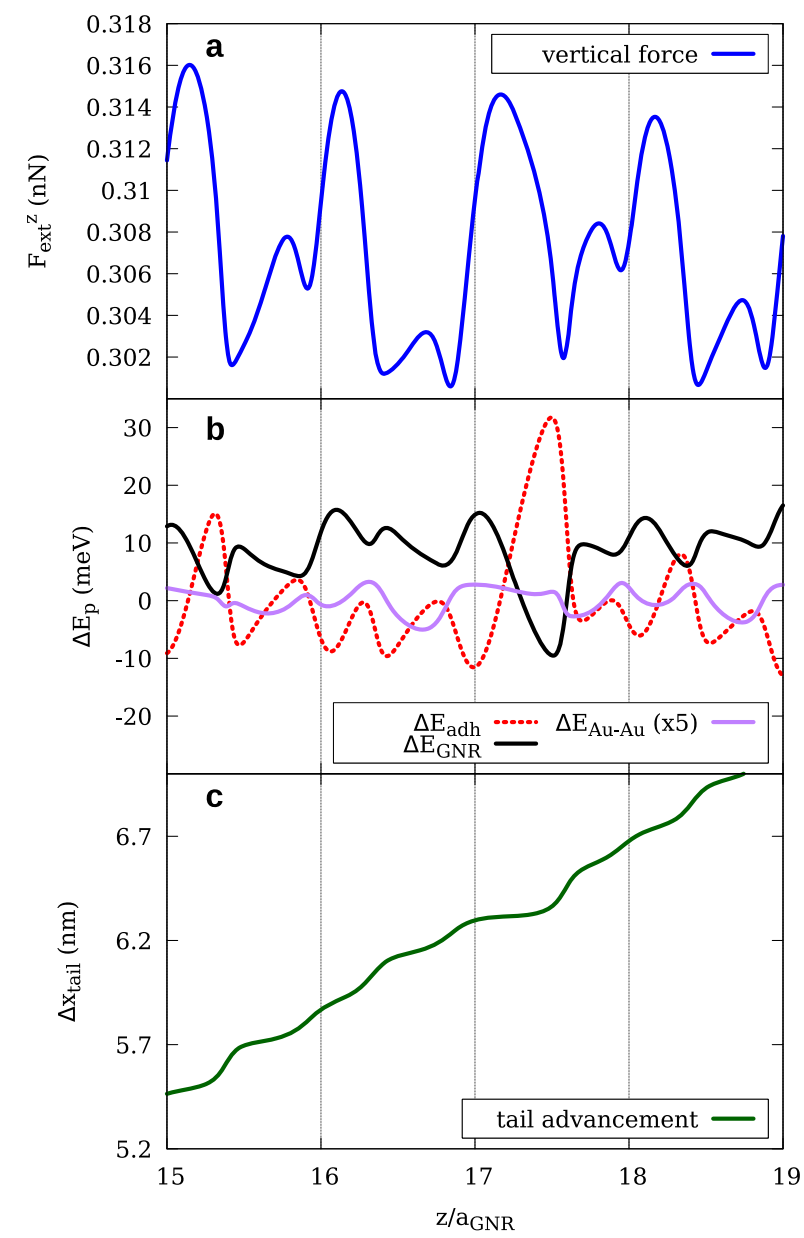

Figure 3. Details of four periods in the simulated detachment dynamics in the steady-state regime. Comparison of (a) the vertical force; $(b)$ the individual potential energy terms-the adhesive energy fluctuations $\Delta E_{\text {adh }}$ (red dotted line), the total variations of the $\mathrm{C}-\mathrm{C}$ intra-GNR potential energy $\Delta E_{\mathrm{GNR}}$ (black solid line), and the $A u-A u$ potential energy $\Delta E_{A u-A u}$ (purple dashed line); (c) the $x$-advancement of the physisorbed GNR body tail. An offset $\Delta=-600 \mathrm{meV}$ has been applied to the $\Delta E_{\mathrm{GNR}}$ curve for better comparison with the other terms.

rows of two- and three-carbon hexagons). Instead, these secondary force derivative features actually arise from the slips, during the detachment dynamics, of the nanoribbon tail, against the lateral corrugation of the gold substrate. The intrinsic incommensurability between $a_{\mathrm{GNR}}=0.42 \mathrm{~nm}$ and $a_{\mathrm{Au}}$ $=0.288 \mathrm{~nm}$ would in principle permit a frictionless, superlubric sliding. However, the trailing end interrupts the GNR$\mathrm{Au}(111)$ moiré pattern, giving rise to an uncompensated region which breaks superlubricity causing a frictional barrier against sliding, capable of causing an unexpected stick-slip. That, together with the role played by the GNR bending elasticity, makes the recorded profile of the lifting force quite complex and rich. More information about this interplay of different length scales can be inferred from the behavior of the individual potential energy terms controlling the system dynamics. We note here that since the external spring drives the GNR away from the substrate at an average constant velocity, we expect the GNR-substrate potential energy to increase linearly in time on average. Likewise, the detachment of the GNR causes the substrate layers to relax around the detachment region, causing the $\mathrm{Au}-\mathrm{Au}$ potential energy to 
decrease linearly with time until (after complete detachment) the Au surface reaches its fully relaxed ground-state energy. We are thus interested in inspecting just the deviations $\Delta E_{\mathrm{adh}}$ and $\Delta E_{\mathrm{Au}-\mathrm{Au}}$ from these linear trends during the detachment procedure. First, we observe that the adhesive energy and bending energy variations for increasing $z$ are anticorrelated. Indeed, during the sticking intervals where the physisorbed tail section remains approximately but not exactly immobile (quasi-horizontal steps of Figure 3c), $\Delta E_{\text {adh }}$ increases, corresponding to a decreasing adhesive energy due to the creeping detachment of one GNR unit cell. In the same time intervals, there is a gain in $\Delta E_{\mathrm{GNR}}$ mainly due to a decrease in the bending cost of the GNR curvature. Once the detachment of one unit cell is complete, the lifted part of the GNR reaches a larger inclination, thus producing an increased lateral $x$ directed force component, driving the physisorbed section of the GNR forward. This force component is generally strong enough to exceed the tenuous lateral Peierls-Nabarro barrier ${ }^{23}$ against sliding of the physisorbed section, thus inducing its advancement, signaled by a slip of the tail, and correspondingly by a drop in $F_{\text {ext }}^{z}(t)$. This dynamics upon strictly vertical lifting is similar to that observed for the case of laterally sliding lifted GNRs. ${ }^{13}$ Since $a_{\mathrm{GNR}} \simeq 1.46 a_{\mathrm{Au}}$, during each main $a_{\mathrm{GNR}}$ period corresponding to detachment of one GNR unit, the simulation exhibits a rather irregular sequence of one or two horizontalslip events, with the corresponding force drops.

Figure 4 shows a frictional loop corresponding to a cycle of detachment/reattachment: the GNR is first lifted up to a nearly detached configuration $\left(z_{\max }=28 \mathrm{~nm}\right)$, and then the spring advancement is stopped and the whole system is relaxed in the lifted configuration. Finally the spring motion is reversed until complete reattachment is achieved. The dynamics in both directions is characterized by similar sequences of attachments/detachments, accompanied by an increase/decrease of the GNR curvature, and followed by one or two slips of the GNR tail. Unsurprisingly, the vertical force is systematically larger in the detachment curve than in reattachment. If the adhesive GNR/substrate potential energy barrier had been the only one to be overcome by the moving spring and if the ribbon motion had been perfectly adiabatic, one might have expected no hysteresis, with the total energy spent for the detachment completely recovered during the reattachment, because the adhesive forces are conservative. In actual fact, in our simulations we observe a significant hysteresis, with two different effects: first, the average vertical force in detachment is larger, and second, there are force peaks (in detachment) and force drops (in reattachment) due to the sudden detachment/ attachment of individual units of the GNR and consequent bending relaxations and tail slips along the upward and downward tracks. These events correspond to intrinsic mechanical instabilities of the system that would, hence, be obtained even in the limit $v_{0} \rightarrow 0$. The difference between the average forces, responsible for the total work of the external force along the up-down cycle, is due to the dissipation in the $T=0$ Langevin thermostat, which is the only term that makes the overall dynamics nonconservative. The dynamical AFM mode that we adopt for our measurements explores steadily a small portion of the histeretic cycle of Figure 4 but at a far smaller speed $\simeq 1 \mu \mathrm{m} / \mathrm{s}$ than in simulations. As a result, the experiment is far closer to the adiabatic regime and finds significant dissipation practically only in connection to the detachments/reattachments of the GNR unit sections. The

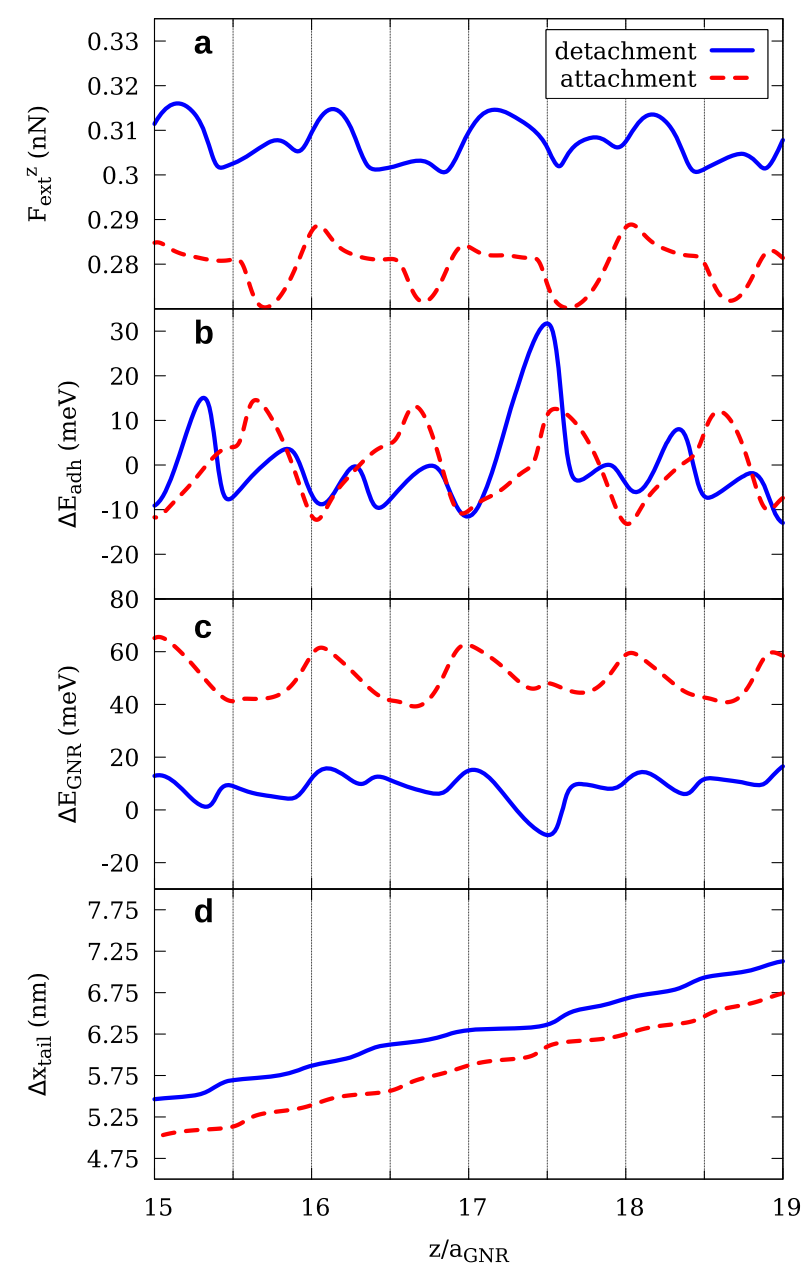

Figure 4. Comparison between the detachment (blue solid lines) and the reattachment (red dashed lines) dynamics of the GNR in four selected periods: (a) pulling force, larger in detachment than in reattachment. The area between the two curves represents the total work done by the moving spring along one detachmentreattachment cycle. (b, c) Main potential energy contributions $\Delta E_{\text {adh }}$ and $\Delta E_{\mathrm{GNR}}$, respectively. An offset $\Delta=-600 \mathrm{meV}$ is applied to the $\Delta E_{\mathrm{GNR}}$ as in Figure 3. (d) Tail $x$-advancement.

resulting experimental energy dissipation curve is reported in the Supporting Information as Figure S1.

As a final observation, Figure $4 c$ shows that the GNR internal energy variation $\Delta E_{\mathrm{GNR}}$ is systematically larger during reattachment than during detachment. This is due to an extra forced bending produced by the spring pushing the GNR down toward the substrate during the reattachment rather than pulling it up as during detachment.

Moiré Pattern Signatures: Detachment at R0. In the previous section, we clarified the main features of the GNR dynamics, determined by the successive detachment of the GNR individual unit sections. By contrast, as pointed out in our previous works, ${ }^{12,13}$ the dependence of the static friction on the size of the GNRs and the sliding dynamics upon lateral pulling was strongly affected by other GNR characteristics, such as the moire-pattern primary periodicity, which gives rise to regions of local registry match/mismatch between the GNR and the substrate.

Surprisingly, no sign of this kind of feature has been observed so far either in the liftoff experiment or in the simulations. We are therefore interested in understanding by 
MD simulations whether this feature could naturally emerge by changing the relative strength between the adhesion of the GNR, which governs the detachment of the GNR unit cells, and the corrugation, which contrasts lateral motions and tunes the moire pattern force contribution. ${ }^{12}$

In our model, one can, for example, reduce the characteristic distance $\sigma$ used in the Lennard-Jones (LJ) potential to describe the GNR/substrate interaction (see the Methods). Figure 5

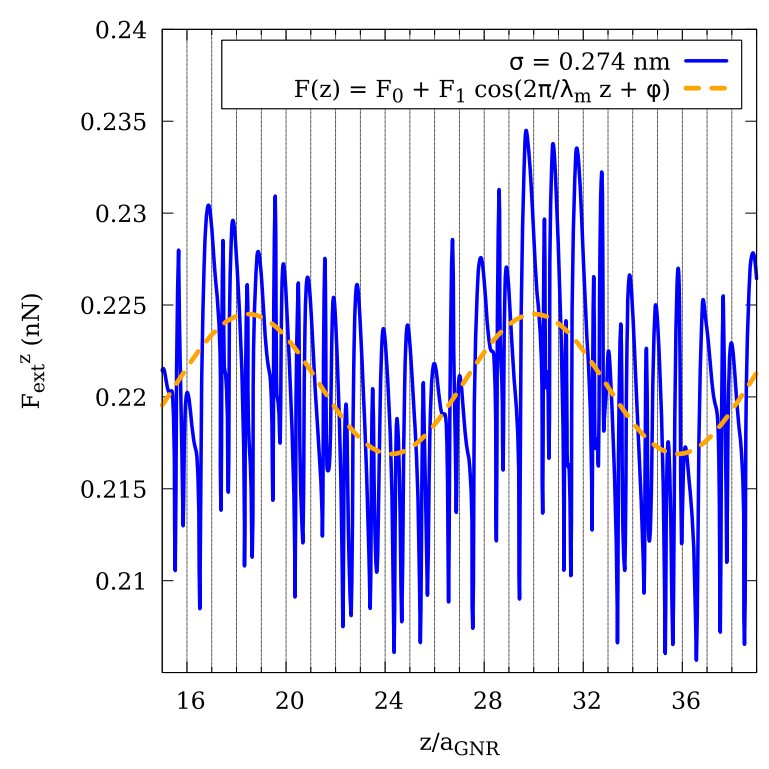

Figure 5. Vertical force profile obtained by artificially modifying the LJ distance $\sigma$ from the regular value $\sigma=0.342 \mathrm{~nm}$ to a smaller one $0.274 \mathrm{~nm}$ (blue solid curve). The fitting function of eq 1 (yellow dashed curve) emphasizes the long-wavelength periodicity, now visible due to the detachment of moire pattern units, at this articially larger interaction strength.

shows the vertical force profile obtained by reducing this distance from the standard $\sigma=0.342 \mathrm{~nm}$ of all previous calculations to $\sigma=0.274 \mathrm{~nm}$. This yields a stronger lateral corrugation, which enhances the contrast between matching and mismatched regions of the moire pattern. ${ }^{12}$ Superimposed on the usual $a_{\mathrm{GNR}}$ periodicity of the GNR unit cells detachment, the lifting force now exhibits a second longwavelength modulation, which can be fitted by a simple sinusoidal function of the form

$$
F(z)=F_{0}+F_{1} \cos \left(\frac{2 \pi}{\lambda_{\mathrm{m}}} z+\varphi\right)
$$

with $F_{0}$ being the average vertical force, $F_{1}$ the amplitude of the force oscillation, $\lambda_{\mathrm{m}}$ its wavelength, and $\varphi$ its phase. The resulting $\lambda_{\mathrm{m}}=4.86 \mathrm{~nm}$ corresponds to the moire pattern wavelength in the R30 alignment. ${ }^{12}$ This is consistent with the subsequent detachment of regions with better adsorption energy, with good local registry between the GNR and the substrate, and regions of poorer adsorption energy with mismatched registry. Since by artificially enhancing the substrate corrugation the moire pattern periodicity becomes evident in the force profile, a similar enhancement could, in principle, occur by choosing a different GNR orientation on the substrate. On $\mathrm{Au}(111)$, all GNR orientations different from R30 are disfavored by the herringbone reconstruction of $\mathrm{Au}(111)$, as mentioned above. $\mathrm{Au}(111)$ could, in principle, be replaced by a different substrate, such as perhaps $\operatorname{Ag}(111)$ or $\mathrm{Cu}(111)$ or others, that are not reconstructed, theoretically permitting different orientations and/or different adhesion energies and different moiré pattern periodicities.

For a qualitative impression of the kind of changes expected, we simulate the detachment of a GNR deposited along the [1 $\overline{1} 0$ ], or R0, alignment, of our hypothetically unreconstructed $\mathrm{Au}(111)$. Both adhesion and corrugation are larger in that GNR alignment, compared to the real R30 alignment addressed above and earlier. ${ }^{12}$

Figure 6 shows the detachment traces from simulations of $\mathrm{GNR} / \mathrm{Au}(111)$ for the $\mathrm{R} 0$ orientation. In this alignment, the

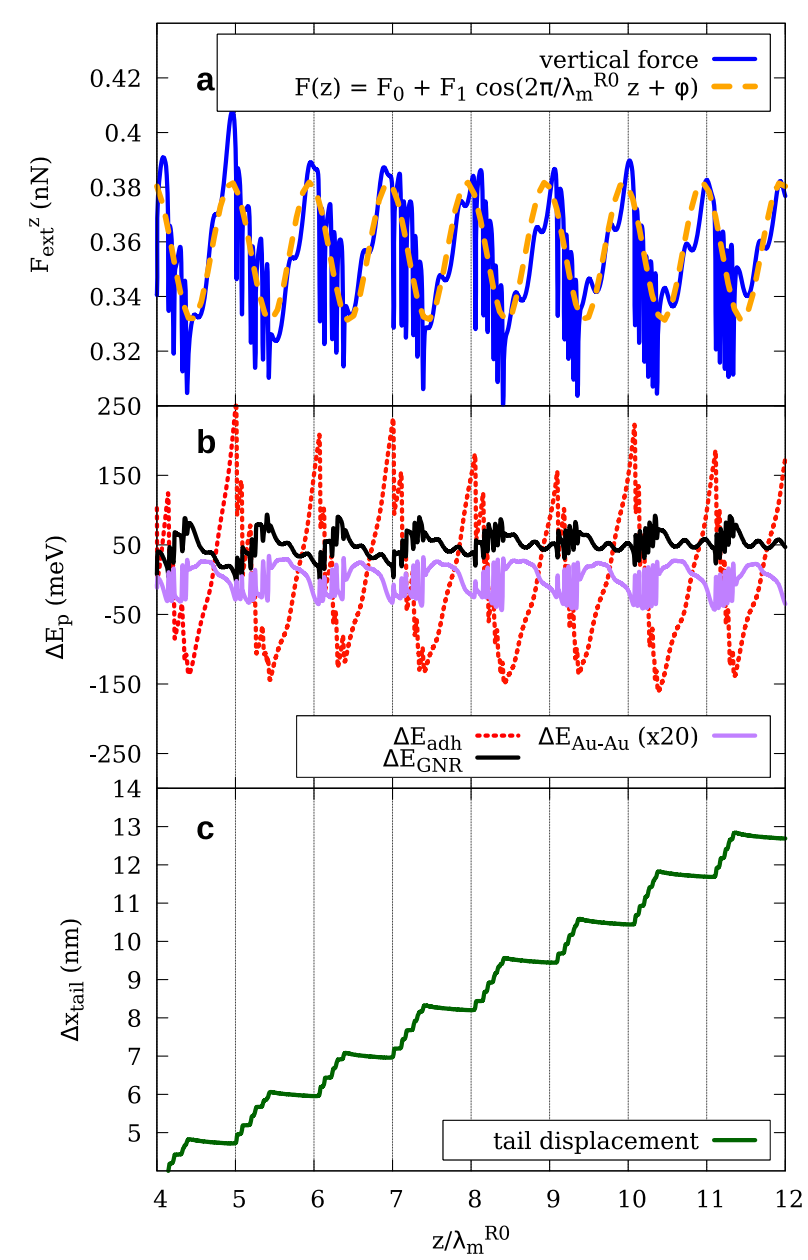

Figure 6. GNR/Au(111) detachment dynamics in the Ro alignment. (a) The fit of the vertical pull-off force with the function of eq 1 (yellow dashed curve) highlights the moire pattern periodicity. (b) Individual components of the total energy. (c) The GNR tail displacement.

substrate periodicity $a_{\mathrm{Au}}=0.499 \mathrm{~nm}$ generates a moiré pattern with primary periodicity $\lambda_{\mathrm{m}}^{\mathrm{R} 0}=1.32 \mathrm{~nm}$ due to its zigzag shape. ${ }^{12}$ With the main periodicity now determined by the moire pattern, the signature of the detachment of the single unit cells of the GNR almost disappears. For this reason we express the quantities of interest as a function of $z / \lambda_{\mathrm{m}}^{\mathrm{R} 0}$. In the R0 alignment, the dynamics consists of successive detachment of entire moire pattern units, as indicated by peaks in the adhesive fluctuations, followed by multiple sliplike advancements of the physisorbed tail, that are accompanied by wide oscillations of the vertical force. Finding no way yet to realize 
the GNR R0 alignment in experiment, otherwise observed in other graphene/metal interfaces, ${ }^{24}$ the status of the results summarized in Figure 6 is that of an interesting theoretical prediction, deserving future experimental investigation.

\section{CONCLUSIONS}

We have described extensive nonequilibrium molecular dynamics simulations of detachment dynamics by vertical lifting of a graphene nanoribbon adsorbed on $\mathrm{Au}(111)$, along with experimental data of the tip-applied force derivative. The results show that the steady state of this vertical dynamics is characterized by the detachments of the individual physisorbed units of the GNR, with the GNR unit cell $0.42 \mathrm{~nm}$ periodicity, in both the experimental tip frequency shift and the simulated vertical-force profiles. These detachments are accompanied by an intermittent stick-slip-like, stop-and-go motion of the tail, which is responsible for extra vertical-force drops that are visible within the main periods.

A qualitatively similar phenomenology is observed during reattachment, where a sequence of unit cell attachments and tail slips still takes place. The frictional loop corresponding to one detachment/reattachment cycle also shows that a nonadiabatic partial detachment/reattachment of the GNR is an intrinsic source of energy dissipation. A non-negligible contribution to the detachment force evolution and to the dissipation comes from the deformability of the Au substrate. Theory overall parallels closely the experimental data, despite quantitative discrepancies due to the impossibility to reproduce in simulations the very low speed as well as the finest adsorption details of the experimental system. Importantly, we have shown that the detachment dynamics can be affected by different competing periodicities depending on the relative strength between the total adhesion and the corrugation of the interface. Enlarging our view, we have explored the simulated detachment for the R0 alignment. Here, the larger corrugation leads to an almost complete disappearance of the GNR unit-cell detachments in favor of moirepattern features, the detachment now involving moiré-pattern periods as entire units. Like the R30 case, the detachment of one complete unit is then followed by sudden multiple slips of the tail.

Further investigations of these observations could involve a theoretical and experimental investigation of this type of nanomanipulation either by changing the GNRs orientation on a possibly unreconstructed gold substrate or possibly by considering different substrates without reconstructions and/or larger corrugations, such as possibly silver, copper, or others.

\section{METHODS}

Experimental Setup. All measurements were performed with a commercially available Omicron low-temperature scanning tunneling microscopy (STM)/atomic force microscopy (AFM) system, operating in ultrahigh vacuum at $4.8 \mathrm{~K}$. We used a tuning fork with a chemically etched tungsten tip as a force sensor. ${ }^{25}$ The resonance frequency and the mechanical quality factor are $23026 \mathrm{~Hz}$ and 19974, respectively. The high cantilever stiffness of $1800 \mathrm{~N} / \mathrm{m}$ realizes a stable operation with a small amplitude of $43 \mathrm{pm} .{ }^{22}$ The frequency shift, caused by the tip-sample interaction, was measured with a commercially available digital phase-locked loop (Nanonis: OC-4 and Zurich Instruments: HF2-LI and HF2-PLL). ${ }^{21}$ For the STM measurement, the bias voltage was applied to the tip while the sample was electronically grounded. The tungsten tip of a tuning fork sensor was sharpened ex situ by focused ion beam milling technique and was then covered in situ with $\mathrm{Au}$ atoms by contacting to the sample surface. A clean gold tip was formed in situ by indenting the $\mathrm{Au}$ sample surface and applying a pulse bias voltage between tip and sample several times. Clean $\mathrm{Au}(111)$ surfaces were prepared in situ by repeated cycles of standard $\mathrm{Ar}^{+}$sputtering $\left(3 \times 10^{-6} \mathrm{mbar}, 1000 \mathrm{eV}\right.$, and $15 \mathrm{~min}$ ) and annealing at $750 \mathrm{~K}$. As precursors to the GNRs synthesis, 10,10'-dibromo-9,9'-dianthryl molecules were deposited on the substrate from a Knudsen cell crucible, heated resistively at $135^{\circ} \mathrm{C}$. Subsequently, the samples were annealed at 200 and $400{ }^{\circ} \mathrm{C}$ to synthesize graphene nanoribbons on $\mathrm{Au}(111){ }^{26,27}$ The STM topographic images were taken in constant current mode. Measured images were partially analyzed using the WSxM software. ${ }^{28}$

Theoretical Modeling. All MD simulations of detachment are performed using the LAMMPS package. ${ }^{29}$ The GNR atoms and the two gold mobile layers obey a $T=0$ dissipative Langevin dynamics, with a damping parameter $\gamma=1.0 \mathrm{ps}^{-1}$, which has been adjusted in order to highlight the stick-slip behavior of the GNR in the steady state. The force fields used to simulate the dynamics of the mobile gold layers and the GNR are the Embedded Atom Method (EAM) potential $^{30,31}$ and the Adaptive Intermolecular Reactive Empirical Bond Order (AIREBO) potential, ${ }^{32}$ respectively.

The $\mathrm{C}-\mathrm{Au}$ and the $\mathrm{H}-\mathrm{Au}$ adhesive interactions are modeled via $(6,12) \mathrm{LJ}$ potentials of the form

$$
V(r)=4 \epsilon\left[\left(\frac{\sigma}{r}\right)^{12}-\left(\frac{\sigma}{r}\right)^{6}\right]
$$

We adopted $\epsilon=8 \mathrm{meV}$ for the $\mathrm{C}-\mathrm{Au}$ interaction and $\epsilon=3.2 \mathrm{meV}$ for the $\mathrm{H}-\mathrm{Au}$ interaction. The adhesive energy per $\mathrm{C}$ atom that we obtain with these values of the LJ amplitudes for flat GNRs on gold is compatible with previous DFT estimates for graphene/metal interfaces ${ }^{33,34}$ and experimental results on graphite/graphite and graphene/silicon contacts. ${ }^{35,36}$ However, we found that the qualitative features of the dynamics are not significantly affected by variations of the LJ parameters in a suitable range of values. These more realistic energies are larger than those adopted in previous work. ${ }^{8,12,13}$ The adsorption distances for carbon and hydrogens are set by a common $\sigma$ $=0.342 \mathrm{~nm}$. This parametrization yields a good match between the shape of the experimental frequency shift profile and the simulated vertical force gradient for the R30 alignment (see Figure $2 b$ ). The same parameters were also used for the simulation of detachment in the R0 alignment.

As discussed above, for the calculations reported in Figure 5, the lateral corrugation was enhanced artificially by changing the value of $\sigma$ to $0.274 \mathrm{~nm}$, while keeping all other parameters unchanged.

\section{ASSOCIATED CONTENT}

\section{Supporting Information}

The Supporting Information is available free of charge on the ACS Publications website at DOI: 10.1021/acsnano.8b07894.

Comparison between the experimental frequency shift trace and the measured energy dissipation per cycle (Figure S1), behavior of the GNR internal energy variation (Figure S2), comparison between the detachment dynamics, in terms of the recorded vertical force profile and the GNR tail advancement (Figure S3), and comparison between the GNR dynamics with a "hard" and a "soft" vertical spring attached to the ribbon short edge (Figure S4) (PDF).

\section{AUTHOR INFORMATION}

\section{Corresponding Authors}

*E-mail: lgigli@sissa.it.

*E-mail: kawai.shigeki@nims.go.jp.

*E-mail: vanossi@sissa.it.

ORCID

Shigeki Kawai: 0000-0003-2128-0120 
Roberto Guerra: 0000-0002-1278-8021

Rémy Pawlak: 0000-0001-8295-7241

Xinliang Feng: 0000-0003-3885-2703

Klaus Müllen: 0000-0001-6630-8786

Pascal Ruffieux: 0000-0001-5729-5354

Roman Fasel: 0000-0002-1553-6487

Andrea Vanossi: 0000-0002-6058-8403

\section{Author Contributions}

L.G. performed the numerical simulations and the analysis of the results. S.K. acquired the experimental measurements. R.G., N.M., E.T., and A.V. guided the interpretation of the experimental and numerical data and the writing of the manuscript. E.M. supervised the experimental study. R.P., X.F., K.M., P.R., and R.F. supported the design of the experimental setup.

Notes

The authors declare no competing financial interest.

\section{ACKNOWLEDGMENTS}

Work in Trieste was carried out by L.G., N.M., E.T., and A.V. under ERC Grant No. 320796 MODPHYSFRICT. S.K. was supported for the experimental part by the Japan Society for the Promotion of Science (JSPS) KAKENHI Grant No. 14K21765; R.P. and E.M. by the Swiss National Science Foundation (SNSF) and the Swiss Nanoscience Institute (SNI). The COST Action MP1303 is gratefully acknowledged.

\section{REFERENCES}

(1) Guo, Z.; Chang, T.; Guo, X.; Gao, H. Gas-Like Adhesion of Two Dimensional Materials onto Solid Surfaces. Sci. Rep. 2017, 7, 159.

(2) Vilhena, J. G.; Gnecco, E.; Pawlak, R.; Moreno-Herrero, F.; Meyer, E.; Perez, R. Stick-Slip Motion of SsDNA over Graphene. J. Phys. Chem. B 2018, 122, 840.

(3) Cao, Z.; Dobrynin, A. Contact Mechanics of Nanoparticles: Pulling Rigid Nanoparticles from Soft, Polymeric Surfaces. Langmuir 2015, 31, 12520 .

(4) Paolicelli, G.; Mougin, K.; Vanossi, A.; Valeri, S. Adhesion, Detachment and Movement of Gold Nanoclusters Induced by Atomic Force Microscopy. J. Phys.: Condens. Matter 2008, 20, 354011.

(5) Kawai, S.; Koch, M.; Gnecco, E.; Sadeghi, A.; Pawlak, R.; Glatzel, T.; Schwarz, J.; Goedecker, S.; Hecht, S.; Baratoff, A.; Grill, L.; Meyer, E. Quantifying The Atomic-Level Mechanics of Single Long Physisorbed Molecular Chains. Proc. Natl. Acad. Sci. U. S. A. 2014, 111,3968

(6) Varini, N.; Vanossi, A.; Guerra, R.; Mandelli, D.; Capozza, R.; Tosatti, E. Static Friction Scaling of Physisorbed Islands: the Key Is in The Edge. Nanoscale 2015, 7, 2093.

(7) Pierno, M.; Bruschi, L.; Mistura, G.; Paolicelli, G.; di Bona, A.; Valeri, S.; Guerra, R.; Vanossi, A.; Tosatti, E. Frictional Transition from Superlubric Islands to Pinned Monolayers. Nat. Nanotechnol. 2015, 10, 714 .

(8) Kawai, S.; Benassi, A.; Gnecco, E.; Söde, H.; Pawlak, R.; Feng, X.; Müllen, K.; Passerone, D.; Pignedoli, C. A.; Ruffieux, P.; Fasel, R.; Meyer, E. Superlubricity of Graphene Nanoribbons on Gold Surfaces. Science 2016, 351, 957.

(9) Pawlak, R.; Ouyang, W.; Filippov, A. E.; Kalikhman-Razvozov, L.; Kawai, S.; Glatzel, T.; Gnecco, E.; Baratoff, A.; Zheng, Q.-S.; Hod, O.; Urbakh, M.; Meyer, E. Single Molecule Tribology: Force Microscopy Manipulation of A Porphyrin Derivative on A Copper Surface. ACS Nano 2016, 10, 713-722.

(10) Guerra, R.; Tosatti, E.; Vanossi, A. Slider Thickness Promotes Lubricity: From 2D Islands to 3D Clusters. Nanoscale 2016, 8, 11108.

(11) Pawlak, R.; Kawai, S.; Meier, T.; Glatzel, T.; Baratoff, A.; Meyer, E. Single-Molecule Manipulation Experiments to Explore Friction and Adhesion. J. Phys. D: Appl. Phys. 2017, 50, 113003.
(12) Gigli, L.; Manini, N.; Benassi, A.; Tosatti, E.; Vanossi, A.; Guerra, R. Graphene Nanoribbons on Gold: Understanding Superlubricity and Edge Effects. 2D Mater. 2017, 4, 045003.

(13) Gigli, L.; Manini, N.; Tosatti, E.; Guerra, R.; Vanossi, A. Lifted Graphene Nanoribbons on Gold: from Atomic Smooth Sliding to Multiple Stick-Slip Regimes. Nanoscale 2018, 10, 2073-2080.

(14) Vanossi, A.; Dietzel, D.; Schirmeisen, A.; Meyer, E.; Pawlak, R.; Glatzel, T.; Kisiel, M.; Kawai, S.; Manini, N. Recent Highlights in Nanoscale and Mesoscale Friction. Beilstein J. Nanotechnol. 2018, 9, 1995.

(15) Baykara, M. Z.; Vazirisereshk, M. R.; Martini, A. Emerging Superlubricity: A Review of The State of The Art and Perspectives on Future Research. Appl. Phys. Rev. 2018, 5, 041102.

(16) Ouyang, W.; Mandelli, D.; Urbakh, M.; Hod, O. Nanoserpents: Graphene Nanoribbons Motion on Two-Dimensional Hexagonal Materials. Nano Lett. 2018, 18, 6009-6016.

(17) Miskin, M.; Sun, C.; Cohen, I.; Dichtel, W. R.; McEuen, P. L. Measuring and Manipulating The Adhesion of Graphene. Nano Lett. 2018, 18, 449-454.

(18) Koch, M.; Li, Z.; Nacci, C.; Kumagai, T.; Franco, I.; Grill, L. How Structural Defects Affect the Mechanical and Electrical Properties of Single Molecular Wires. Phys. Rev. Lett. 2018, 121, 047701.

(19) Cai, J.; Ruffieux, P.; Jaafar, R.; Bieri, M.; Braun, T.; Blankenburg, S.; Muoth, M.; Seitsonen, A.; Saleh, M.; Feng, X.; Müllen, K.; Fasel, R. Atomically Precise Bottom-Up Fabrication of Graphene Nanoribbons. Nature 2010, 466, 470.

(20) Talirz, L.; Ruffieux, P.; Fasel, R. OnSurface Synthesis of Atomically Precise Graphene Nanoribbons. Adv. Mater. 2016, 28, 6222.

(21) Albrecht, T. R.; Grütter, P.; Horne, D.; Rugar, D. Frequency Modulation Detection Using High-Q Cantilevers for Enhanced Force Microscope Sensitivity. J. Appl. Phys. 1991, 69, 668.

(22) Giessibl, F. J. Advances in Atomic Force Microscopy. Rev. Mod. Phys. 2003, 75, 949-983.

(23) Floría, L.; Mazo, J. Dissipative Dynamics of The FrenkelKontorova Model. Adv. Phys. 1996, 45, 505.

(24) Wofford, J. M.; Starodub, E.; Walter, A. L.; Nie, S.; Bostwick, A.; Bartelt, N. C.; Thürmer, K.; Rotenberg, E.; McCarty, K. F.; Dubon, O. D. Extraordinary Epitaxial Alignment of Graphene Islands on $\mathrm{Au}(111)$. New J. Phys. 2012, 14, 053008.

(25) Giessibl, F. J. High-Speed Force Sensor for Force Microscopy and Profilometry Utilizing a Quartz Tuning Fork. Appl. Phys. Lett. 1998, 73, 3956.

(26) Ruffieux, P.; Wang, S.; Yang, B.; Sánchez-Sánchez, C.; Liu, J.; Dienel, T.; Talirz, L.; Shinde, P.; Pignedoli, C. A.; Passerone, D.; Dumslaff, T.; Feng, X.; Müllen, K.; Fasel, R. On-Surface Synthesis of Graphene Nanoribbons with Zigzag Edge Topology. Nature 2016, $531,489$.

(27) Jacobberger, R. M.; Kiraly, B.; Fortin-Deschenes, M.; Levesque, P. L.; McElhinny, K. M.; Brady, G. J.; Delgado, R. R.; Roy, S. S.; Mannix, A.; Lagally, M. G.; Evans, P. G.; Desjardins, P.; Martel, R.; Hersam, M. C.; Guisinger, N. P.; Arnold, M. S. Direct Oriented Growth of Armchair Graphene Nanoribbons on Germanium. Nat. Commun. 2015, 6, 8006.

(28) Horcas, I.; Fernandez, R.; Gomez-Rodriguez, J.; Colchero, J.; Gomez-Herrero, J.; Baro, A. WSXM: A Software for Scanning Probe Microscopy and a Tool for Nanotechnology. Rev. Sci. Instrum. 2007, 78,013705

(29) Plimpton, S. Fast Parallel Algorithms for Short-Range Molecular Dynamics. J. Comput. Phys. 1995, 117, 1.

(30) Daw, M. S.; Baskes, M. I. Embedded-Atom Method: Derivation and Application to Impurities, Surfaces, and Other Defects in Metals. Phys. Rev. B: Condens. Matter Mater. Phys. 1984, 29, 6443.

(31) Finnis, M. W.; Sinclair, J. E. A Simple Empirical N-body Potential for Transition Metals. Philos. Mag. A 1984, 50, 45.

(32) Brenner, D. W.; Shenderova, O. A.; Harrison, J. A.; Stuart, S. J.; Ni, B.; Sinnott, S. B. A Second-Generation Reactive Empirical 
Bondorder (REBO) Potential Energy Expression for Hydrocarbons. J.

Phys.: Condens. Matter 2002, 14, 783.

(33) Giovannetti, G.; Khomyakov, P. A.; Brocks, G.; Karpan, V. M.; van den Brink, J.; Kelly, P. J. Doping Graphene with Metal Contacts. Phys. Rev. Lett. 2008, 101, 026803.

(34) Vanin, M.; Mortensen, J. J.; Kelkkanen, A. K.; Garcia-Lastra, J. M.; Thygesen, K. S.; Jacobsen, K. W. Graphene on etals: A Van Der Waals Density Functional Study. Phys. Rev. B: Condens. Matter Mater. Phys. 2010, 81, 081408.

(35) Koren, E.; Lörtscher, E.; Rawlings, C.; Knoll, A. W.; Duerig, U. Adhesion and Friction in Mesoscopic Graphite Contacts. Science 2015, 348, 679 .

(36) Zong, Z.; Chen, C.-L.; Dokmeci, M. R.; Wan, K.-T. Direct Measurement of Graphene Adhesion on Silicon Surface by Intercalation of Nanoparticles. J. Appl. Phys. 2010, 107, 026104. 